\title{
Maconha e gravidez: síndrome da hiperêmese por canabinoide - Relato de caso
}

\author{
Marijuana and pregnancy: cannabis hyperemesis syndrome - Case report \\ Daniel Luis Tittonel Justi', João Baptista Laurito Jr.2. Alexandre Quelho Comandule³, Eglinara Silva Morton ${ }^{4}$
}

\begin{abstract}
Palavras-chave
Síndrome de hiperêmese por canabinoide, maconha e banho quente, síndrome dos vômitos cíclicos, hiperêmese gravídica.
\end{abstract}

\section{Keywords}

Cannabinoid hyperemesis syndrome, marijuana and hot shower, cyclic vomiting syndrome, hyperemesis gravidarum.

\section{RESUMO}

Os autores relatam um caso da enfermaria psiquiátrica do Hospital Geral Santa Casa de Misericórdia de Sorocaba-SP. Paciente gestante, de 19 anos, usuária crônica de maconha que apresenta náuseas e vômitos intensos não responsivos aos antieméticos, associados a dor abdominal, agitação psicomotora e hábito compulsivo de tomar banhos quentes para alívio dos sintomas, quadro que caracteriza a SHC. Trata-se de uma síndrome rara, com efeito paradoxalmente emetogênico da maconha. É subdiagnosticada e relacionada ao uso crônico e intenso de maconha. Destacamos que não foi encontrada descrição de caso na literatura brasileira. A conclusão deste relato reporta a importância do reconhecimento e categorização da síndrome e sua correlação com a dependência de maconha, pois o tratamento implica a suspensão do uso de maconha, tratamento da dependência, além da prevenção de suas complicações clínicas.

\section{ABSTRACT}

The authors report a case from the psychiatric department of a general hospital (Santa Casa de Misericordia at Sorocaba city, São Paulo, Brazil). A patient, 19-year-old, pregnant woman, marijuana chronic user, presenting abdominal pain, psychomotor agitation, unmanageable nausea, severe vomiting, not responsive to antiemetic drugs, associated with compulsive hot water showering. Cannabinoid hyperemesis syndrome is sub-diagnosed and rare, characterized by those three symptoms: heavy marijuana use, vomiting and repeated hot showering. There is no prior report in Brazilian medical literature. This paper aims to emphasize the importance of recognizing and categorizing this syndrome and its correlation to marijuana use. Main treatment is supportive care, withdrawing substance and supportive carries to suspension of marijuana use as well as clinical complications prevention.

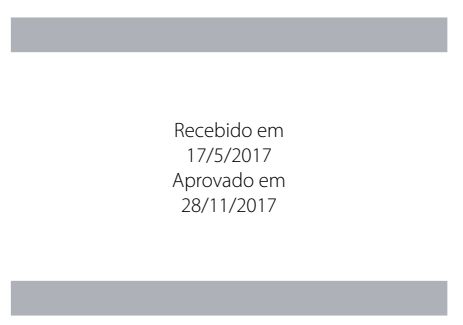

DOI: $10.1590 / 0047-2085000000185$

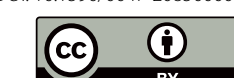

1 Prefeitura Municipal de Sorocaba, Enfermaria Psiquiátrica da Santa Casa de Misericórdia de Sorocaba.

2 Universidade Estadual de Campinas, Faculdade de Ciências Médicas (Unicamp/FCM); Prefeitura Municipal de Sorocaba, Programa de Residência em Psiquiatria; Enfermaria Psiquiátrica da Santa Casa de Sorocaba.

3 Universidade Estadual de Campinas, Faculdade de Ciências Médicas (Unicamp/FCM); Enfermaria Psiquiátrica da Santa Casa de Sorocaba. 4 Prefeitura Municipal de Sorocaba, Programa de Residência em Psiquiatria; Enfermaria Psiquiátrica da Santa Casa de Sorocaba; Universidade Federal do Estado do Rio de Janeiro (UNIRIO); Universidade de São Paulo (USP). 


\section{INTRODUÇÃO}

A maconha é a terceira droga recreacional e a primeira ilícita mais consumida no mundo e no Brasil ${ }^{1-9}$, apresentando prevalência de 7,5\% de uso na gravidez ${ }^{8,10}$.

Após a legalização da maconha e a permissão para seu uso medicinal e recreacional, seu consumo tem crescido nos Estados Unidos ${ }^{2,7,11}$. Esse crescimento traz novos quadros patológicos ${ }^{9}$, entre eles a síndrome da hiperêmese por canabinoide $(\mathrm{SHC})^{2,5,11}$.

Desde o primeiro caso de SHC, relatado em 2004 na Austráliaa ${ }^{5,12}$, sua incidência aumentou no mundo ${ }^{2,5,13}$. Na literatura científica, foram encontrados 90 artigos no banco de dados PubMed, entre eles seis de pacientes grávidas. Não foi encontrado no Brasil relato de caso publicado, fato que motivou a publicação deste artigo.

A SHC surge em decorrência do consumo acentuado e crônico (pelo menos um ano) de maconha $2,6,8,11,13,14$ e tem como sintoma característico a compulsão por banho quente ${ }^{7}$.

A síndrome é constituída pelas fases prodrômica, êmese e recuperação. Na fase prodrômica, há ansiedade, agitação, sintomas autonômicos com sudorese, rubor e sede. Na fase de êmese, ocorrem náuseas e vômitos incoercíveis, sem resposta a antieméticos convencionais, além de compulsão por banho quente ${ }^{7}$. E na fase de recuperação, ocorre a resolução dos sintomas, que na maioria dos pacientes se verifica entre 24 e 48 horas. Essa resolução ocorreria em manejo conservador e com a cessação do consumo canábico. Contudo, essa fase pode se estender até um mês ${ }^{1,2,5}$.

A demora para o diagnóstico se deve ao desconhecimento de sua ocorrência e de sua fisiopatologia, resultando em lacuna diagnóstica de meses a anos $1,5,7,7,11$. Dentre as complicações, ainda desconhecidas, destacam-se a insuficiência renal aguda ${ }^{11,14,15}$, esofagite erosiva ${ }^{14}$, alcalose metabólica e hipocalemia ${ }^{8}$.

\section{CASO CLÍNICO}

C.V.S., de 19 anos, gestante de 32 semanas, G3P1A1, foi internada na enfermaria de psiquiatria do Hospital Santa Casa de Sorocaba por apresentar comportamento alterado, ansiedade, crises de choro, inapetência e história de múltiplos episódios de vômitos, principalmente matutinos, de moderada quantidade, intratáveis com antieméticos tradicionais, além de dor abdominal. Esses sintomas só se atenuavam quando a paciente rastejava pelo chão ou com banhos muito quentes, o que, segundo sua mãe, se repetia várias vezes ao dia, por horas, com risco de queimaduras.

"Doutora, ela só quer ficar no chuveiro muito quente e tenho medo que se queime, porque faz isso por horas e às vezes chega a tomar 10 banhos por dia", diz a genitora em entrevista.
A paciente apresentou histórico de cinco episódios semelhantes no último ano, quando buscava frequentemente serviços de emergência clínica. Essa era sua terceira internação psiquiátrica (ver Tabela 1). Relatava histórico de uso de maconha iniciado há quatro anos, com consumo de cinco a quinze cigarros por dia, o que configurava padrão de dependência.

Durante a internação, manteve pensamento coerente e conexo, porém estava muito ansiosa, com certa puerilidade, inapetente e com importantes episódios de vômitos, de moderada quantidade, alguns de conteúdo bilioso, que não cessavam com antieméticos intravenosos (metoclopramida, ondansetrona). Apresentava comportamento bizarro de se arrastar e correr, sempre inquieta, tinha que se manter em movimento o tempo todo e chorava constantemente. Destacava seu intrigante comportamento compulsivo por banhos quentes. Em um desses banhos, a paciente descreveu que a água acalmava e organizava seus pensamentos.

"Por favor, doutora, me deixa ir pro chuveiro, eu imploro! Eu sei que no chuveiro eu melhoro", disse a paciente em entrevista.

A paciente foi avaliada pela obstetrícia, que confirmou ser a gravidez tópica de 32 semanas e que o feto estava saudável. Realizou exames laboratoriais com resultados normais e, devido à incapacidade de manter a ingesta alimentar e a importância dos vômitos, foi administrada hidratação intravenosa.

Tendo em vista a evolução favorável de melhora dos sintomas, inicialmente pela cessação do comportamento bizarro de rastejar-se e, por último, da hiperêmese e redução dos banhos, além da preocupação com sua gravidez em ambiente de enfermaria, ela recebeu alta médica após três dias de internação.

O diagnóstico de SHC foi sugerido após busca na literatura científica sobre relatos que descreviam as queixas da paciente, principalmente compulsão por banhos quentes, hiperêmese e abuso de maconha. Tal diagnóstico foi comunicado imediatamente à paciente e à sua genitora, bem como foram fornecidos esclarecimentos sobre sua síndrome e a necessidade de continuar abstinente.

Após a alta, a paciente manteve-se abstinente de maconha, sem apresentar a sintomatologia descrita, mantendo seguimento regular no Centro de Atenção Psicossocial (CAPS) com um dos autores.

O bebê nasceu de parto cesáreo com 35 semanas apresentando leve desconforto respiratório, mas com rápida resolução. As medicações foram suspensas de forma gradual e, até a resposta da submissão deste artigo, ela se manteve abstinente, com remissão completa da psicopatologia e sem uso de medicações. A paciente visitou a enfermaria e levou suas filhas. Ela comentou que o chuveiro hoje é seu alerta para não ter recaída e concordou em participar deste relato assinando o Termo de Consentimento Livre e Esclarecido. 
Tabela 1. Resumo das internações da paciente

\begin{tabular}{|c|c|c|c|}
\hline & 1a Internação & 2a Internação & 3a internação \\
\hline Idade gestacional & 18 semanas & 26 semanas & 32 semanas \\
\hline CID-10 - alta & $\mathrm{F} 60.4, \mathrm{~F} 42.1$ & $\mathrm{~F} 44.7, \mathrm{~F} 12.2, \mathrm{~F} 42.1$ & $\mathrm{~F} 12.2, \mathrm{~F} 42.2$ \\
\hline Tempo de internação & 6 dias & 5 dias & 3 dias \\
\hline Medicação na alta & $\begin{array}{c}\text { Sertralina } 100 \mathrm{mg} \text {, haloperidol } 5 \mathrm{mg}, \\
\text { clonazepam } 2 \mathrm{mg}\end{array}$ & Sertralina $100 \mathrm{mg}$, haloperidol $5 \mathrm{mg}$ & $\begin{array}{l}\text { Sertralina } 150 \mathrm{mg} \text {, haloperidol } 5 \mathrm{mg} \text {, } \\
\text { levomepromazina } 50 \mathrm{mg}\end{array}$ \\
\hline Encaminhamento para rede extra-hospitalar & $\begin{array}{c}\text { CAPS II } \\
\text { (Centro de Atenção Psicossocial) }\end{array}$ & $\begin{array}{c}\text { CAPS II } \\
\text { (Centro de Atenção Psicossocial) }\end{array}$ & $\begin{array}{c}\text { CAPS AD } \\
\text { (Centro de Atenção Psicossocial Álcool e Drogas) }\end{array}$ \\
\hline
\end{tabular}

F60.4 - Transtorno de Personalidade Histriônica; F42.1 - Transtorno Obsessivo-Compulsivo com predominância de comportamentos compulsivos; F44.7 - Transtorno dissociativo misto; F12.2 - Transtornos mentais e comportamentais devidos ao uso de canabinoides - Síndrome de dependência; F42.2 - Transtorno Obsessivo-Compulsivo, forma mista, com ideias obsessivas e comportamentos compulsivos.

\section{DISCUSSÃO}

A SHC tem sintomatologia pouco específica e resolução espontânea dos casos após a cessação do uso da maconha. Pela falta de informação entre profissionais da saúde, a SHC é subdiagnosticada. Concordante com a literatura, também neste relato, houve atraso e dificuldade diagnóstica pelo CAPS e pela enfermaria psiquiátrica. Somente após a terceira internação, concluiu-se o diagnóstico. Na Tabela 2, de Simonetto et al., os sintomas foram compilados como critérios sugestivos de diagnóstico 1,89,11,12. Esses critérios são compatíveis com a sintomatologia do relato do caso.

Tabela 2. Fatores diagnósticos da síndrome de hiperêmese por canabinoide

\begin{tabular}{l}
\hline Critério Essencial \\
\hline Consumo de maconha cronicamente \\
\hline Critérios Maiores \\
\hline Vômitos e náuseas cíclicas severas \\
Resolução dos sintomas com a cessação da maconha \\
Melhora dos sintomas com banho de água quente \\
Dor abdominal (epigástrica ou periumbilical) \\
Consumo semanal da maconha \\
\hline Critérios Menores \\
\hline Idade abaixo de 50 anos \\
Perda de peso acima de 5 kg \\
Predominância dos sintomas pela manhã \\
Hábitos intestinais normais \\
Testes negativos laboratoriais, radiológicos e endoscópicos \\
\hline Fonte: Simonetto et al!!
\end{tabular}

A fisiopatologia dessa síndrome é pouco conhecida e os estudos relacionam a reação paradoxal tóxica ao consumo regular da maconha, que em consumo leve tem efeitos antieméticos, mas em uso severo leva à hiperêmese $e^{2,6,8,11,13}$.

A maconha tem entre seus compostos o delta-9-tetra-hidro-canabinol (THC), o canabidiol (CBD) e o canabigerol $(\mathrm{CBG})^{13}$. A literatura científica menciona a ação, dose-dependência e meia-vida do THC, CBD e CBG nos receptores de canabinoides CB1 presentes no trato gastrointestinal e cerebral (cerebelo, glândula pituitária, hipotálamo, gânglios da base e hipocampo $)^{5,8}$. No trato gastrointestinal, os componentes da maconha provocam a diminuição do esvaziamento gástrico $^{5,11,13}$, da secreção gástrica, da peristalse ${ }^{7}$, do tônus do esfíncter esofágico inferior ${ }^{5,13}$ e causam inapetência, náusea, vômito"1 e dor visceral5,11, enquanto no cérebro controla, principalmente, a termorregulação $15,12,13$.

O efeito tóxico do THC depositado na gordura corporal seria o responsável pelos sintomas intestinais agindo em receptores $\mathrm{CB} 1$ 1,2,5,7,11,12. Associado a essa toxicologia, há o polimorfismo genético relacionado ao citocromo P450 de alguns indivíduos, que pode alterar o metabolismo dos canabinoides, resultando em níveis excessivos de canabinoides pró-eméticos ou metabólitos emetogênicos ${ }^{5,8,11}$. Além disso, o processo de reintoxicação ocorre quando há lipólise em resposta ao estresse ou privação alimentar ${ }^{5,11}$.

Alguns autores relacionam a melhora dos sintomas eméticos com a redistribuição do sangue do intestino para a pele, produzida pelo banho com água quente ${ }^{2,7,14}$. Foi observada melhora significativa dos sintomas quando a paciente ficava sob o chuveiro.

Não há consenso sobre o tratamento desse quadro, e as condutas variam entre diversos autores. Os tratamentos incluiriam cuidados de suporte com fluidos intravenosos, correção de distúrbios eletrolíticos ${ }^{7,11,13}$, haloperido|6,11, narcóticos $^{5,11}$, inibidores de bomba de próton e manutenção de banhos quentes 5 . A cessação do consumo da maconha é conduta consensual e essencial no tratamento da SHC 1,2,8,11-14, uma vez que os sintomas normalmente cessam em poucos dias de abstinência ${ }^{5,7}$. A paciente fazia uso de sertralina 100 mg, cuja dose foi ajustada para 150 mg. O fármaco haloperidol foi mantido para promover resposta antiemética e controle da agitação, além de se promover a abstinência da maconha como principal fator resolutivo da sintomatologia e das constantes internações.

Entre os principais diagnósticos diferenciais encontrados na literatura científica, encontram-se a síndrome do vômito cíclico (SVC), o vômito psicogênico (VP) 2,5 e a hiperêmese gravídica $(H G)^{7,8}$. Diferentemente da SHC, na SVC há história pessoal de migrânea e estressor psicológico, e no VP há relação com a depressão ou transtorno conversivo ${ }^{2,5}$. No caso deste artigo, o diagnóstico de transtorno de personalidade 
histriônica (TPH) foi sugerido, pela constante queixa da paciente em ser atendida em suas exigências durante as crises de choro, seguida pelo comportamento infantilizado e desejo de banho quente ou de arrastar-se pelo chão. A HG é um diagnóstico diferencial importante, entretanto, nesse diagnóstico há melhora dos sintomas com antieméticos e não há na história o uso de maconha, tampouco comportamento relacionado ao banho quente como fator de melhora?. Suspeitou-se ainda de transtorno obsessivo-compulsivo, pela constante procura pelo chuveiro para alívio da ansiedade.

\section{CONCLUSÃO}

É importante não se minimizarem a dependência e uso nocivo de maconha como fatores primordiais para o correto diagnóstico da SHC. A síndrome SHC com seus sintomas característicos descritos ainda não foi incluída no DSM-V, tampouco na CID-10, o que contribui para a sua subnotificação. Incentivar sua categorização e reconhecimento e prospectar novos estudos é essencial para melhor entendimento da incidência, prevalência e fisiopatologia da SHC, bem como para compreender os mecanismos de ação neuroquímicos dos diversos componentes da maconha e o papel de cada um nos efeitos recreativos, médicos, psicológicos e nocivos relacionados ao seu uso.

\section{CONTRIBUIÇÕES INDIVIDUAIS}

Daniel Luis Tittonel Justi - Realizou levantamento bibliográfico, leitura dos artigos, redação e formatação do artigo.

Alexandre Quelho Comandule - Realizou levantamento bibliográfico e revisão do artigo e auxiliou na sua formatação.

Eglinara Silva Morton - Realizou levantamento, leitura, redação, formatação e revisão do artigo.

João Baptista Laurito Jr. - Realizou leitura, redação e formatação.

\section{CONFLITOS DE INTERESSE E DECLARAÇÕES DE SUPORTES FINANCEIROS}

Os autores não possuem conflitos de interesse ou suporte financeiro a serem declarados.

\section{AGRADECIMENTOS}

Agradecemos ao Dr. Wellington Alencar Carvalho, pela colaboração em nos enviar o primeiro artigo sobre a síndrome e pelo incentivo à publicação; ao Dr. André Vedovato e à Drielle Morton, pelo apoio e orientações; à Dra. Tatyane Coló Yamagute e à Dra. Sanja Lukic pela tradução do resumo para a língua inglesa.

\section{REFERÊNCIAS}

1. Simonetto DA, Oxentenko AS, Herman ML, Szostek JH. Cannabinoid hyperemesis: a case series of 98 patients. Mayo Clin Proc. 2012;87(2):114-9.

2. Lu ML, Agito MD. Cannabinoid hyperemesis syndrome: Marijuana is both antiemetic and proemetic. Cleve Clin J Med. 2015;82(7):429-34.

3. Jungerman FS, Menezes PR, Pinsky I, Zaleski M, Caetano R, Laranjeira R. Prevalence of cannabis use in Brazil: data from the I Brazilian National Alcohol Survey (BNAS). Addict Behav. 2010;35(3):190-3.

4. Ribeiro HL, Renno Jr. J, Demarque R, Cavalsan JP, Rocha R, Cantilino A, et al. Efeito do consumo de cannabis na gravidez e no período pós-parto. Rev Debates Psiquiatr. 2016:16-24.

5. Galli JA, Sawaya RA, Friedenberg FK. Cannabinoid hyperemesis syndrome. Curr Drug Abuse Rev. 2011;4(4):241-9.

6. Hickey JL, Witsil JC, Mycyk MB. Haloperidol for treatment of cannabinoid hyperemesis syndrome. Am J Emerg Med. 2013;31(6):1003.e5-6.

7. Alaniz VI, Liss J, Metz TD, Stickrath E. Cannabinoid hyperemesis syndrome: a cause of refractory nausea and vomiting in pregnancy. Obstet Gynecol. 2015;125(6):1484-6.

8. Hermes-Laufer J, Puppo LD, Inn I, Troillet FX, Kherad 0. Cannabinoid hyperemesis syndrome: a case report of cyclic severe hyperemesis and abdominal pain with long-term cannabis use. Case Rep Gastrointest Med. 2016;2016:2815901.

9. Schmid SM, Lapaire 0, Huang DJ, Jürgens FE, Güth U. Cannabinoid hyperemesis syndrome: an underreported entity causing nausea and vomiting of pregnancy. Arch Gynecol Obstet. 2011;284(5):1095-7.

10. Barbosa TD, Miranda MP, Nunes GF, Schutte TS, Santos K, Monteiro DLM. Manifestações do uso da maconha e opiáceos durante a gravidez. Femina. 2011;39(7):403-7.

11. Sorensen CJ, DeSanto K, Borgelt L, Phillips KT, Monte AA. Cannabinoid hyperemesis syndrome: diagnosis, pathophysiology, and treatment - a systematic review. J Med Toxicol. 2017;13(1):71-87.

12. Allen JH, de Moore GM, Heddle R, Twartz JC. Cannabinoid hyperemesis: cyclical hyperemesis in association with chronic cannabis abuse. Gut. 2004;53(11):1566-70.

13. Ramadurai S, Gopalan S, Arthur P. Cannabinoid hyperemesis syndrome as an unusual cause of cyclic vomiting. Indian J Psychiatry. 201;58(2):234-5.

14. Srihari P, Liu M, Punzell S, Shebak SS, Rea WS. Cannabinoid hyperemesis syndrome associated with compulsive showering and acute kidney injury. Prim Care Companion CNS Disord. 2016;18(1)

15. Habboushe J, Sedor J. Cannabinoid hyperemesis acute renal failure: a common sequela of cannabinoid hyperemesis syndrome. Am J Emerg Med. 2014;32(6):690.e1-2. 